\title{
Spectrum Continuity and Contiguity based Dedicated Protection for Flexible Optical Networks
}

André V. S. Xavier, Raul C. Almeida Jr., Joaquim F. Martins-Filho, Federal University of Pernambuco, e-mail: jfmf@ufpe.br

Daniel A. R. Chaves and Carmelo J. A. Bastos-Filho

University of Pernambuco, e-mail: darc@ecomp.poli.br, carmelofilho@ieee.org

\begin{abstract}
Elastic optical networks (EONs) have been pointed out as a promising candidate for transporting data with high transmission rates in adaptive optical networks. Consequently, the occurrence of a failure in a simple element may cause the interruption of various services. Survival mechanisms have been crucial to try to overcome the problems created by possible failures. In this paper, we propose a new dedicated path protection mechanism for link-failure survivability in EONs, referred to as Spectrum Continuity and Contiguity based Dedicated Protection (SCCDP). SCCDP tries to avoid the trap topology problem in search for a link-disjoint route pair. In addition, the calculation of the link-disjoint routes is based on modified version of the Dijkstra's algorithm, referred to as Spectrum Continuity and Contiguity based Shortest Path (SCCSP), which chooses the pair of routes according to the end-to-end spectrum continuity and contiguity. The performance of the proposed algorithm is compared to other well-known algorithms in the Literature. Two different network topologies are used in our simulations for comparison purposes. Our proposal achieved better results concerning blocking probability.
\end{abstract}

Index Terms - Dedicated protection, Path protection, Elastic optical networks, Single failure, Survivability.

\section{INTRODUCTION}

The Internet traffic has grown in recent years due to the increased number of users and new data-demanding applications, such as video on demand and streaming services in real time. Wavelength division multiplexing (WDM) optical networks have been applied in transport networks to supply these requirements of traffic. These systems are capable of handling the channels through wavelength division multiplexing (WDM), where each WDM channel have been traditionally filled up with transmission rates of 10,40 or $100 \mathrm{~Gb} / \mathrm{s}$ [1], [2].

The use of a fixed spectral band for every WDM channel independently of the traffic intensity and modulation format used generates an inefficient use of the spectrum for scenarios with heterogeneous services. Since the end of last decade, there has been increasing interest in the investigation of new optical networking architectures without the fixed grid employed in WDM optical networks. In such new architectures, the management layer and network elements support flexible bandwidth for the channels, which are adjusted according to the characteristics of the network requests. Elastic optical networks (EONs) have been proposed as a promising candidate for high transmission rates in adaptive optical transport networks [3]. Some recently 
developed technologies have been proposed to provide the desirable flexibility in the optical domain for this new type of network architecture, such as transponders with variable bandwidth, wavelength-selective switches (WSSs) with variable bandwidth, efficient fiber-optic transmission technologies (like coherent optical orthogonal frequency division multiplexing, CO-OFDM) [4], Nyquist-WDM and dynamic optical arbitrary waveform generation (OAWG) [5], [6]. This adaptability maximizes the efficiency in the use of spectrum resources by using a finer spectral unit, called frequency slot. Besides, new switching technologies enable that lightpaths be provisioned with multiple frequency slots.

Two constraints in spectrum management in EONs are the spectrum contiguity and spectrum continuity. In the spectrum contiguity, if a call request needs two or more frequency slots, these frequency slots must be adjacent in the optical spectrum. On the other hand, spectrum continuity means that the same frequency slots assigned to a call request must be used in all links of the route found to implement this call request [7]. Analogous to the routing and wavelength assignment (RWA) process in WDM optical networks, the development of efficient routing and spectrum assignment (RSA) algorithms is an important challenge for EONs. These algorithms have a direct influence on how the occupancy of the frequency slots turn up among the network links and, as a consequence, it has a significant impact on the network performance. RSA consists of finding a route between the source and destination nodes with a required number of contiguous and continuous frequency slots on the links along this route [8]. If it is assumed that one may choose, for each call request, an appropriate modulation format among some available ones, the RSA process is referred to as routing, modulation level and spectrum assignment (RMLSA) [9].

Another important factor in EONs is to provide survivability mechanisms against failures. The occurrence of a single failure in one or more network elements can lead to a massive loss of information. The survivability mechanisms are commonly divided into protection and restoration techniques. Protection techniques are based on providing resource reservation to guarantee the connection protection after a failure. Protection techniques can be divided into the categories of dedicated path protection (DPP) and shared path protection (SPP). In DPP, a dedicated link-disjoint backup lightpath is configured to protect each working lightpath on the network. In the SPP, the backup lightpath can be shared among other backup routes [10].

On the other hand, restoration techniques are based on the best effort recovery mechanism and are triggered by the occurrence of the failure. When a connection is affected by a failure, the restoration algorithm tries to find a new lightpath to restore the interrupted connection. Restoration techniques are more efficient regarding network resource utilization, but it does not guarantee an available lightpath to restore the affected connection. The three most important methods to perform restoration in a network are path restoration, subpath restoration, and link restoration. In the path restoration, one tries to find a new lightpath between the source and destination nodes. In the link restoration, one attempts to find a new lightpath from the adjacent nodes to the failure. Finally, in the subpath restoration, one tries to find a lightpath from the previous node to the failure until the destination node, reusing a portion of the existing lightpath [10].

DDP techniques ensure a fast and guaranteed traffic recovery from single failures as well as an easier implementation. On the other hand, it presents a higher resource utilization ratio when compared to both shared protection and restoration techniques [11], [12]. In this paper, we focus on the application of the DPP method for EONs. We propose a novel algorithm to protect connections against a single link failure. We also consider physical layer impairments. We name the proposed algorithm as Spectrum Continuity and Contiguity based 
Dedicated Protection (SCCDP). SCCDP finds a link-disjoint route pair based on the end-to-end spectrum continuity and contiguity. The remainder of the paper is organized as follows. In Section II, we present some related works and some solutions in the literature, whereas, in Section III, we present a description of the proposed SCCDP algorithm. Section IV details the simulation setup and the parameters used in the simulations. In Section V, we present and discuss the simulation results, and in Section VI, we present our conclusions.

\section{RELATED WORKS}

In this section, we review some solutions in the literature for solving the DPP problem in EONs. The simplest approach for tackling the DPP problem in WDM optical networks attempted to use Dijkstra's or Bellman-Ford's algorithm [13] in two steps. The first step is to calculate the working route in the network topology, and the links of this route are temporarily removed from the network. In the second phase, the protection route is determined. This approach is easily implemented. However, after the temporary removal of the links used in the working path, the network may be broken into two disconnected portions which would make impossible to discover a backup route. Such scenario may occur even when two link-disjoint paths exist in the network. These network topologies are commonly referred to as trap topologies. Suurballe et al. [14] proposed an algorithm to solve the problem of finding link-disjoint pairs on trap topologies. The Suurballe's algorithm tries to avoid the critical links in the trap topology, allowing the algorithm to find a link-disjoint route pair if it exists.

Takagi et al. [15] proposed some algorithms to solve the RSA problem considering dedicated and shared path protection in EONs. The developed algorithms are based on an integer linear programming (ILP) model. This model considers the distance-adaptive constraint. These proposed algorithms are compared to WDM scenario regarding spectrum efficiency. The achieved results show a reduction around 30\% in required resources.

Klinkowski et al. [16] proposed a genetic algorithm (GA) to solve the problem of RSA with dedicated path protection in an EON with static traffic demand and subject to single-link failures. Other graph-based algorithms were used for the sake of comparison with the proposed algorithm regarding spectrum efficiency.

Vizcaíno et al. [17] proposed heuristics to investigate power consumption and cost model problems in both WDM and EON architectures. Due to the importance of survivability in optical networks, the paper considers and evaluates both dedicated path protection and shared path protection. The results present the potential energy efficiency improvements that can be obtained by using EONs.

Walkowiak et al. [18] proposed an ILP model to solve the offline problem of routing and spectrum assignment (RSA) with dedicated path protection (DPP). The formulation uses a modified version of the Tabu Search method to provide near optimal solutions.

Shen et al. [19] developed ILP models to minimize the number of link frequency slots used and the required spare capacity. Shared path protection (SPP) and dedicated path protection (DPP) techniques were considered. Both techniques were analyzed under the scenarios with and without tunable transponders. All models were compared to each other in three network topologies. The results show that the SPP techniques require lower spare capacity when compared to the DPP techniques.

Seb J. Savory [20] proposed heuristics to investigate congestion-aware routing problem in nonlinear elastic optical networks. The algorithm uses a cost function based on congestion to evaluate the links in the network as shown in Eq. (1). 


$$
W_{i, j}=\frac{d_{i, j}}{\eta_{i, j}},
$$

where $W_{i, j}$ is the cost associated to the link between nodes $i$ and $j$, which is given by the ratio of the link length $\left(d_{i, j}\right)$ and the fraction of spectrum that is available on the link $\left(\eta_{i, j}\right)$. After evaluating the link's cost, Dijkstra Algorithm is used to find the route to attend the connection request. This heuristics may be easily adapted to solve the DPP problem in elastic optical networks.

\section{SCCDP ALGORITHM}

In this paper, we propose a new dedicated path protection algorithm to deal with single-link failure in EONs. This new proposal is named spectrum continuity and contiguity based dedicated protection (SCCDP). The SCCDP algorithm finds a link-disjoint route pair in the network considering the spectrum continuity and contiguity information during the process. The SCCDP pseudocode is presented in Algorithm 1. It applies some steps proposed by Suurballe et al. [14] to find a link-disjoint route pair and to deal with the trap topology problem (lines 5-9). SCCDP algorithm determines a route $P l$ with spectrum continuity and contiguity using the $S C S P$ routing algorithm in $T$ (line 2). In line 5, the $S C C D P$ algorithm removes temporarily the uplinks of $P 1$ (or reverses the links of $P 1$ ) from $T$ and finds a new route, $P 2$. If $P 2$ is not found, the SCCDP algorithm rejects the connection request, as described in line 18. Otherwise, the algorithm compares and removes the shared links between $P 1$ and $P 2$. These shared links must be avoided during the calculation of both link-disjoint working and protection routes (lines 7-9), since the use of these shared links may split the network into disconnected parts [13], as previously discussed. In lines 2, 3, 7 and 9, the SCSP algorithm is responsible for finding a route with the lowest cost considering end-to-end spectrum continuity and contiguity.

Xavier et al. [21] proposed a routing algorithm that considers the spectrum continuity and contiguity of the frequency slots during the routing process. This algorithm is referred to as Spectrum Continuity based Shortest Path (SCSP). The SCSP algorithm uses a modified version of Dijkstra's algorithm, where the information about both continuity and contiguity of the route formed from the origin node until an intermediate node in the Dijkstra process is combined with the spectrum availability of each link leaving the node to form the cost of each of these links. The pseudocode of the SCSP algorithm is presented in Algorithm 2. The vector continuity is initialized in line 2. This vector stores the frequency slots availability information from the source node until the other nodes according to the route with the lowest cost. The vectors cost and father and the list $Q$ are initialized in lines 2-6. The frequency slots are all considered as available (value = " 1 ") in the source node, and this information is stored in vector continuity (line 8). In line 11, the neighbor nodes of the node $u$ are visited, and the vectors continuity $[u]$ and $D$ are compared slot by slot (lines 13-21). Then, the resultant vector of this comparison, $R$, is used to evaluate the cost of the link as shown in line 22. The cost function, $f(d(u, v), R, k)$, is calculated based on two variables. The first variable, $x_{u, v}$, considers the normalized physical link length, as shown in (2). 


\section{ALGORITHM 1. SCCDP ALGORITHM PSEUDOCODE.}

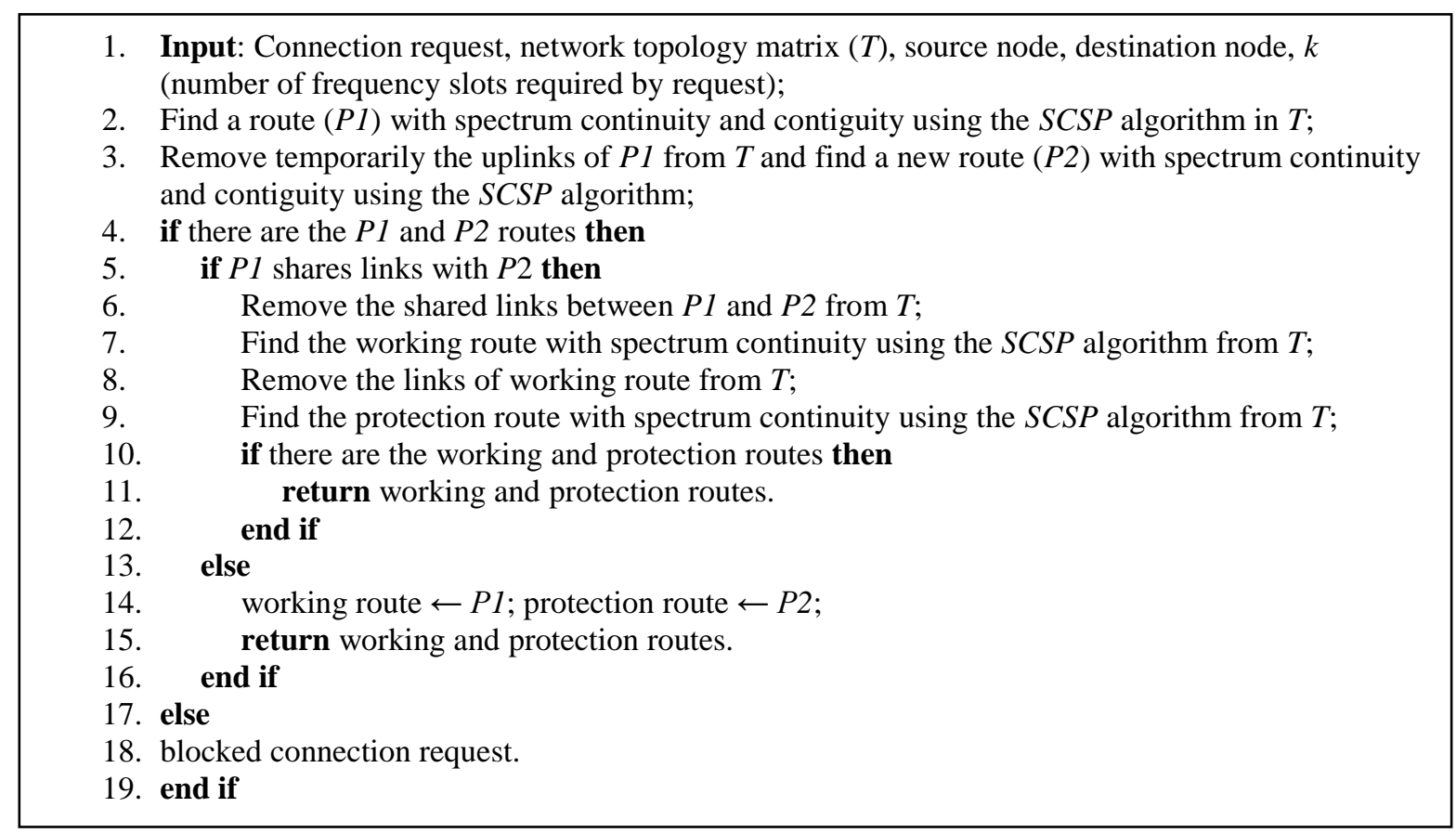




\section{ALGORITHM 2. SCSP ALGORITHM PSEUDOCODE.}

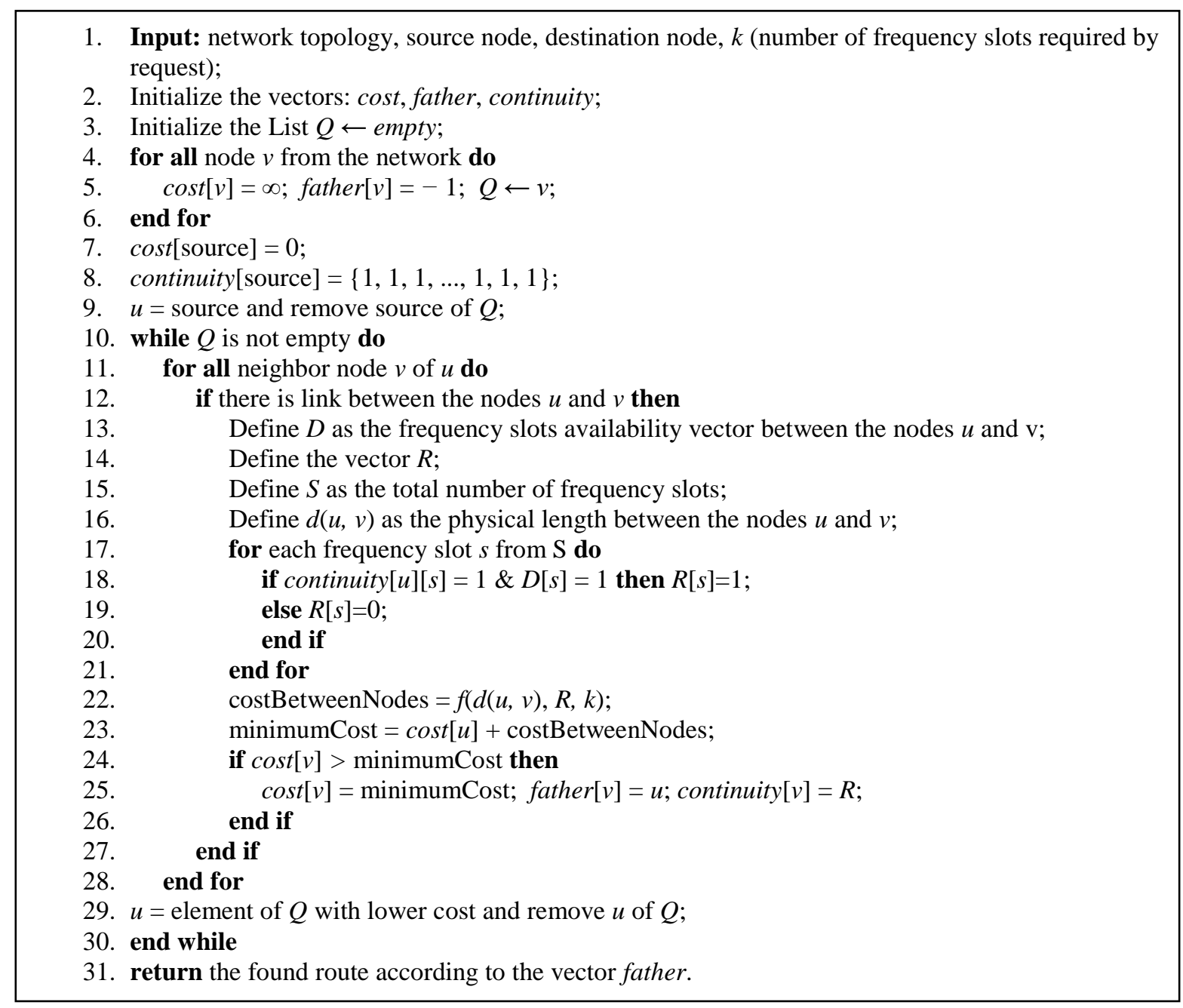

$$
x_{u, v}=\frac{d_{u, v}}{d_{\max }},
$$

in which $d_{u, v}$ is the link length between nodes $u$ and $v$ and $d_{\max }$ is the maximum link length in the network. The parameter $x_{u, v}$ contributes to reduce the path request blocking occurrences due to physical layer constraints. The second parameter, $y_{R, k}$, considers the information regarding frequency slots contiguity, as well as the spectrum continuity from source node until the currently analyzed node, this information of the spectrum continuity is stored in the algorithm during the analysis of the network link, the parameter $y_{R, k}$ is shown in Eq. (3). The variable $y_{R, k}$ is responsible for reducing the path request blocking occurrences due to lack of resources.

$$
y_{R, k}=\frac{1}{s_{R, k}+1},
$$

in which $S_{R, k}$ accounts for is the frequency slots contiguity. $S_{R, k}$ is obtained by the number of possible manners of assigning the current $k$-slot request in the available spectrum of the vector $R$, as proposed in [21]. 
The cost function ( $f$ ) of SCSP algorithm using the two variables is shown in Eq. (4).

$$
f\left(x_{u, v}, y_{R, k}\right)=\frac{d_{u, v}}{d_{\max }}+\frac{1}{s_{R, k}+1} .
$$

Another cost function for the SCSP algorithm using only the spectrum availability information is presented in Eq. (5). Both cost functions are used in the simulations of proposed algorithm.

$$
f\left(y_{R, k}\right)=\frac{1}{s_{R, k}+1} .
$$

\section{SimULATION SETUP}

The SIMTON simulation tool [22] was adapted to evaluate the blocking probability of dedicated path protection algorithms in survivable EON scenarios. For each network simulation, a set of 100,000 calls is generated with source-destination node pairs selected by using a uniform distribution. The call request arrival process is characterized as a Poisson process and the time duration for each established lightpath follows an Exponential distribution. Moreover, each call request has its bit rate demand defined randomly with uniform distribution. The allowed bit rates are 40,100, 200 and 400 Gbps. Fig. 1 shows the flowchart of the dedicated path protection, modulation level and spectrum assignment algorithms employed in the network simulations. For each call request, the DPP algorithm tries to find a link-disjoint route pair. If there is not a link-disjoint route pair for this call request, the request is blocked. Otherwise, the process tries to choose an appropriate modulation format for each route, starting from the most efficient modulation format. One can observe that the modulation format determines the number of necessary contiguous frequency slots for each call. If either working or backup route has no contiguous and continuous frequency slots to attend the call request, the call is blocked. In case there are free slots, the quality of transmission (QoT) is evaluated. If either working or backup routes has QoT below the acceptance threshold, the second most efficient modulation format is tested, and this process continues until the most robust modulation format is checked. In all simulations, the working and protection lightpaths may be assigned with different modulation formats.

The performance indicator used to evaluate the DPP algorithm in this paper is the network blocking probability. The network blocking probability is obtained as the ratio of the number of blocked call requests to the total number of call requests. For the call request to be accepted in the network, the dedicated protection algorithm has to find a pair of link-disjoint routes, both with available resources (contiguous and continuous frequency slots) and enough QoT. The physical layer impairments considered in this paper are ASE (Amplified Spontaneous Emission) noise and ASE saturation in the amplifiers [23].

The configuration of the link containing the devices is presented in [23]. We used a pre-amplifier and a booster for each optical link for the loss compensation. On certain cases, lightpaths passing through multiple optical links can accumulate ASE, and this can mitigate the OSNR of the lightpath. 


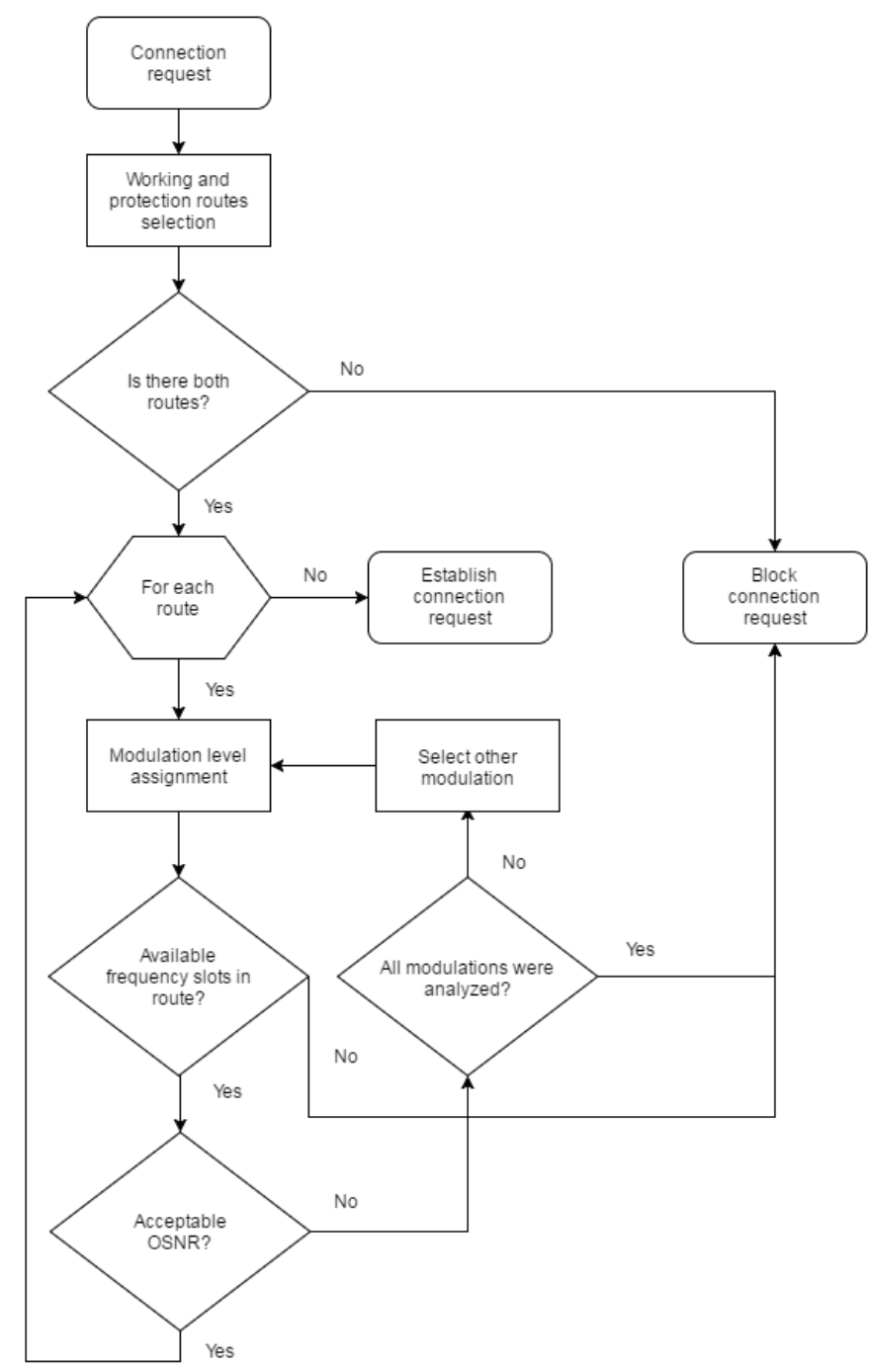

Fig. 1. Flowchart of the dedicated path protection, modulation level and spectrum assignment algorithms employed in the network simulations.

The modulation formats allowed in our simulations are QPSK, 8-QAM, 16-QAM and 32-QAM. The optical parameters used in the simulations are shown in Table I.

In our simulations, we used two different network topologies, as shown in Figs. 2 and 3, respectively. The network topologies 1 and 2 have an average link distance of $46.66 \mathrm{~km}$ and $103.04 \mathrm{~km}$, respectively. The links are bidirectional, i.e., they are formed by a pair of optical fibers, one for each direction. We assume that both working and backup lightpaths are bidirectional, each of which is routed through the same links (with optical fibers in the opposite direction) and no spectrum conversion capability is available at intermediate nodes. The required OSNR (OSNR $\left.{ }_{\text {th }}\right)$ for each modulation format depends on the transmission bit rate and the SNR per bit of the modulation format. The SNR per bit of the modulation format and the equation used to calculate the $\mathrm{OSNR}_{\mathrm{th}}$ can be found in [24]. Table II shows the number of frequency slots for each combination of modulation level and transmission bit rate assuming frequency slots of $12.5 \mathrm{GHz}$. Table III shows the OSNR th (in $\mathrm{dB}$ ) for each combination of modulation level and transmission bit rate. In all simulations, we have assumed a guard band of $12.5 \mathrm{GHz}$. 
TABLE I. DEFAULT SIMULATION PARAMETERS.

\begin{tabular}{ccc}
\hline Parameter & Value & Definition \\
\hline$P_{\text {laser }}$ & $0 \mathrm{dBm}$ & Output transmission power \\
$P_{\text {sat }}$ & 11 & Ampl. output sat. power \\
OSNR $R_{\text {in }}$ & 40 & Input OSNR \\
$S$ & 320 & Number of frequency slots \\
$\Delta f$ & $12.5 \mathrm{GHz}$ & Frequency slot spacing \\
$\alpha$ & $0.2 \mathrm{~dB} / \mathrm{km}$ & Fiber loss coefficient \\
$L_{\text {Mux }}$ & $3 \mathrm{~dB}$ & Multiplexer loss \\
$L_{\text {Demux }}$ & $3 \mathrm{~dB}$ & Demultiplexer loss \\
$L_{\text {Switch }}$ & $10 \mathrm{~dB}$ & Optical switch loss \\
$\mathrm{NF}$ & $5.5 \mathrm{~dB}$ & Amplifier noise figure
\end{tabular}

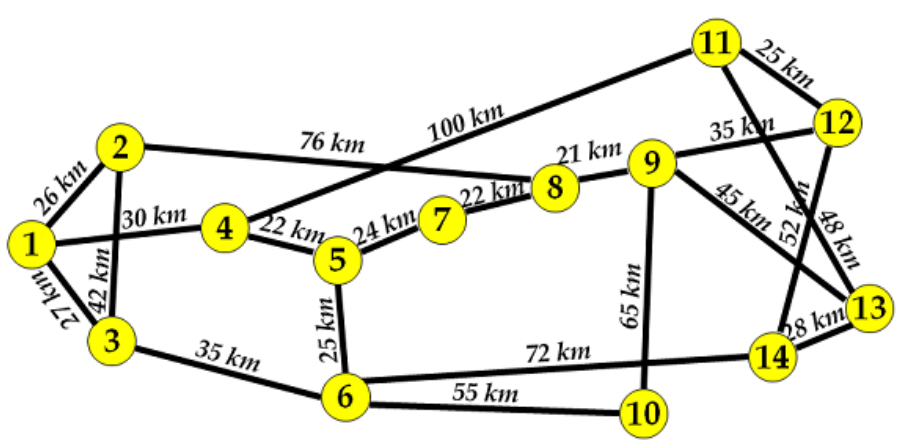

Fig. 2. Network topology 1.

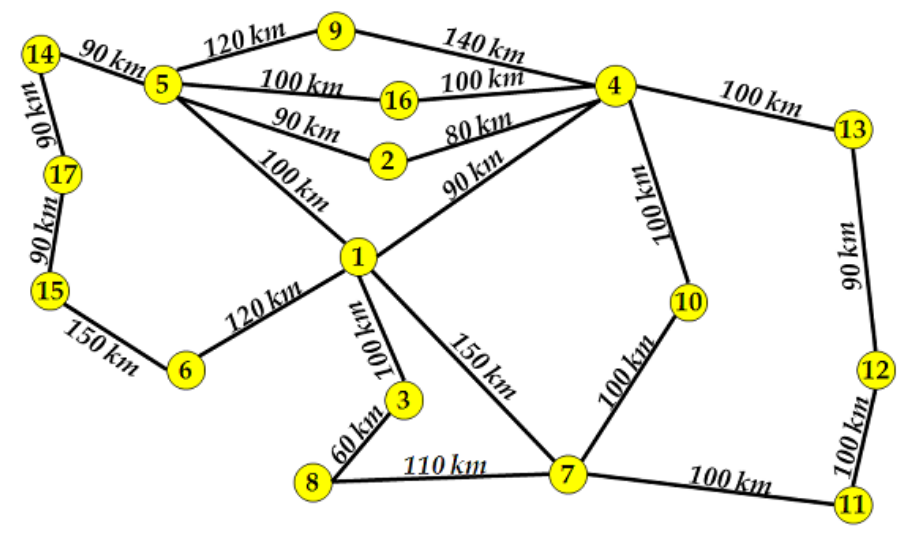

Fig. 3. Network topology 2. 
TABLE II. NUMBER OF FREQUENCY SLOTS FOR EACH COMBINATION OF MODULATION LEVEL AND TRANSMISSION BIT RATE.

\begin{tabular}{ccccc}
\hline Modulation/Bit rate & 40 Gbit/s & 100 Gbit/s & 200 Gbit/s & 400 Gbit/s \\
\hline QPSK & 2 & 4 & 8 & 16 \\
8-QAM & 2 & 3 & 6 & 11 \\
16-QAM & 1 & 2 & 4 & 8 \\
32-QAM & 1 & 2 & 4 & 7 \\
\hline
\end{tabular}

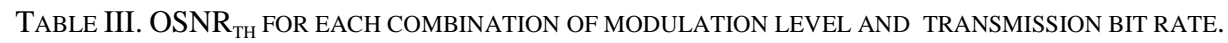

\begin{tabular}{ccccc}
\hline Modulation/Bit rate & 40 Gbit/s & 100 Gbit/s & 200 Gbit/s & 400 Gbit/s \\
\hline QPSK & 8.83 & 12.81 & 15.82 & 18.83 \\
8-QAM & 12.04 & 16.02 & 19.03 & 22.04 \\
16-QAM & 12.56 & 16.54 & 19.55 & 22.56 \\
32-QAM & 14.61 & 18.59 & 21.60 & 24.61 \\
\hline
\end{tabular}

As the SCCDP algorithm needs information about the required number of frequency slots to calculate the routes before determining the modulation format, we assume that the algorithm considers the number of frequency slots used by the most robust of the modulation formats (QPSK), which is the worst case. However, after the routing process, the $S C C D P$ algorithm may select another more efficient modulation format to attend the connection request.

Variables $x_{u, v}$ and $y_{R, k}$ defined in Section III, are used in the SCCDP algorithm, which yields two distinct versions: $S C C D P(\mathrm{y})$, which searches for the link-disjoint route pair based on the information of the spectrum continuity and contiguity, so that the physical distance does not influence the routing decision process, and the second version, $\operatorname{SCCDP}(\mathrm{x}, \mathrm{y})$, which uses information about both the link physical distance and the spectrum continuity and contiguity during the search for the link-disjoint route pair.

We used two versions of the Suurballe's algorithm for the sake of comparison. The first release is the classical algorithm, which uses the link length $\left(x_{u, v}\right)$ as the link cost function. The other version is an adaptation of Suurballe algorithm to use information about the frequency slots contiguity $\left(y_{R, k}\right)$ as a parameter to evaluate the network links' costs. Such versions are referred to as Suurballe(x) and Suurballe(y), respectively. Another algorithm adapted to solve the DPP problem was based on the routing algorithm proposed by Savory [20]. This algorithm is referred to as congestion-aware dedicated protection (CADP). The CADP algorithm evaluates the links of the network by using Eq. (1). Then, the Dijkstra's algorithm is run twice to find working and protection routes. After finding the pair of routes for the DPP process, the spectrum assignment is done using the First-Fit $(F F)$ algorithm [25][26]. In this algorithm, all frequency slots are labeled with numbers in a sequential order, and the $F F$ algorithm searches for the first available spectrum range with the necessary consecutive number of slots considering the ascending order of the frequency slot index.

\section{Simulation RESUlts}

In this section, we present results regarding the network blocking probability of Suurballe(x), Suurballe(y), $C A D P, S C C D P(\mathrm{y})$ and $S C C D P(\mathrm{x}, \mathrm{y})$ algorithms, all of them using the $F F$ spectrum assignment.

Figures 4 and 5 show the comparison of the path request blocking probability as a function of the network load obtained after 30 different runs of the simulation process for all investigated DPP algorithms on the network topologies 1 and 2, respectively. The symbols stand for the average blocking probability achieved by 
each algorithm, and the error bars represent a confidence interval of 95\%. In some cases, the error bars are smaller than the symbols size, and therefore cannot be perceived in the graphs. In Fig. 4, it can be observed that $S C C D P(\mathrm{y})$ outperformed any other algorithms, which can be explained by the fact that $S C C D P(\mathrm{y})$ algorithm used the spectrum continuity and contiguity information of the frequency slots to better select the pair of routes to meet the requests in the network more efficiently than Suurballe(y), CADP and SCCDP(x,y) algorithms. Although the $\operatorname{SCCDP}(\mathrm{x}, \mathrm{y})$ algorithm considers the spectrum continuity during the routing process, the introduction of the variable $x_{u, v}$ in the algorithm makes the pair of shortest routes be chosen more frequently, which may reduce the chance of selecting alternative routes with higher spectrum availability which would usually present additional chance of having end-to-end free spectrum resource. Notice that the majority of nodes in network topology 1 has a connectivity equal to 3, i.e., there are at most three pairs of link-disjoint routes for each node pair. If some algorithm always uses the same two of the three existing routes to attend the call requests for a given source and destination pair, eventually, the network will not be able to provide resources to attend new demands.

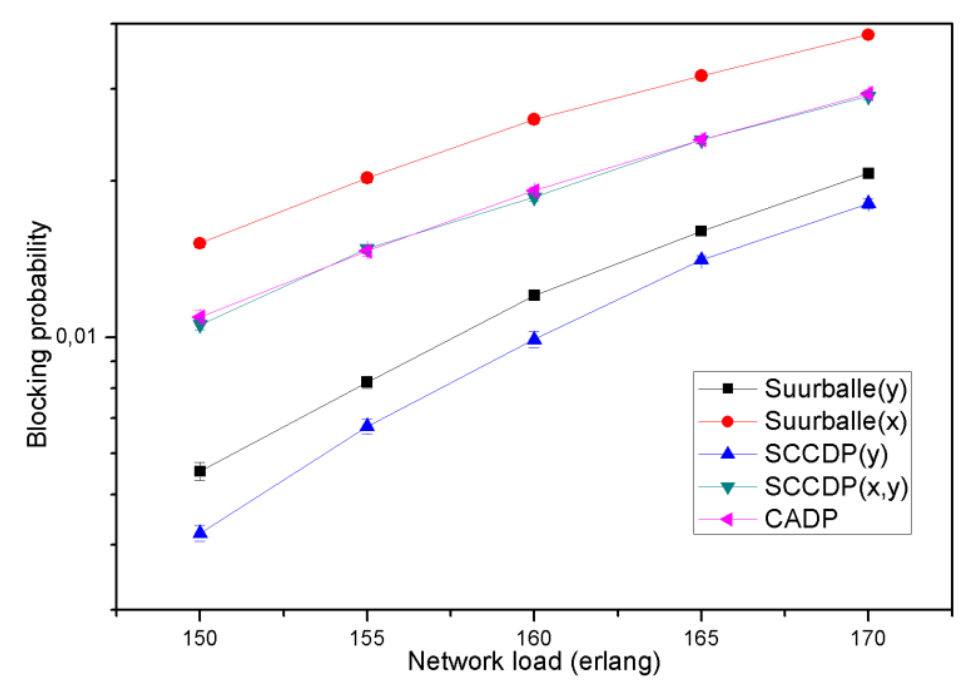

Fig. 4. Comparison of the blocking probability as a function of the network load after 30 different runs and its margin of error at 95\% confidence interval for all investigated DPP algorithms applied to Network Topology 1.

In Fig. 4, the Suurballe(y) algorithm obtained the second best performance. This occurs because the algorithm may analyze and evaluate the state of the links regarding frequency slots contiguity, which results in an adaptive dynamic routing process, although it cannot infer the information about the end-to-end frequency slot continuity. Regarding the $C A D P$ algorithm, it weights the links in the network as a function of both the physical length and the proportion of the total spectrum that is still available. Since the CADP algorithm does not consider information about the continuity and contiguity of frequency slots, it is expected that the algorithm in some cases does not choose efficient routes with enough available spectrum resources to attend the connection requests.

It is important to emphasize that, in both analyzed topologies, two of the network characteristics that affect the performance of the DPP algorithms are the node degree and the distribution of links in the network topology. The node degree is the number of links connected to the node, which impacts the number of alternative linkdisjoint routes that a routing mechanism can find. These characteristics have an impact on obtaining the pair of routes with end-to-end frequency slots continuity. Other important network features are the number of available 
frequency slots and the possible modulation formats.

In Fig. 5, all algorithms obtained similar performance in network topology 2. This occurs because, in such topology, the blocking due to the lack of quality of transmission is predominant. The $\operatorname{SCCDP}(\mathrm{y})$ algorithm chooses its routes based only on frequency slots availability, which returns solutions, in some case, that do not present the necessary quality of transmission. Another factor that contributes for the algorithms to obtain similar performance is related to the constraints in Network Topology 2. The fact that its majority of nodes has connectivity equal to 2 results that the algorithms find, for most of the source-destination nodes in the network, just one pair of link-disjoint routes.

It is important to notice that our target blocking probability for operation in dynamic networks is $1 \%$. Also, dedicated protection implies in the assignment of two lightpaths for each call. Besides, one can observe from the topologies that some of the links concentrate traffic and, as a consequence, they represent bottlenecks for the lightpath assignment process. A call request is denied if one of the two lightpaths can not be implemented due to unavailable resources or inappropriate Quality of Transmission. This is a hard constraint and causes an increase in the blocking probability, leading to high blocking probability for lower network loads.

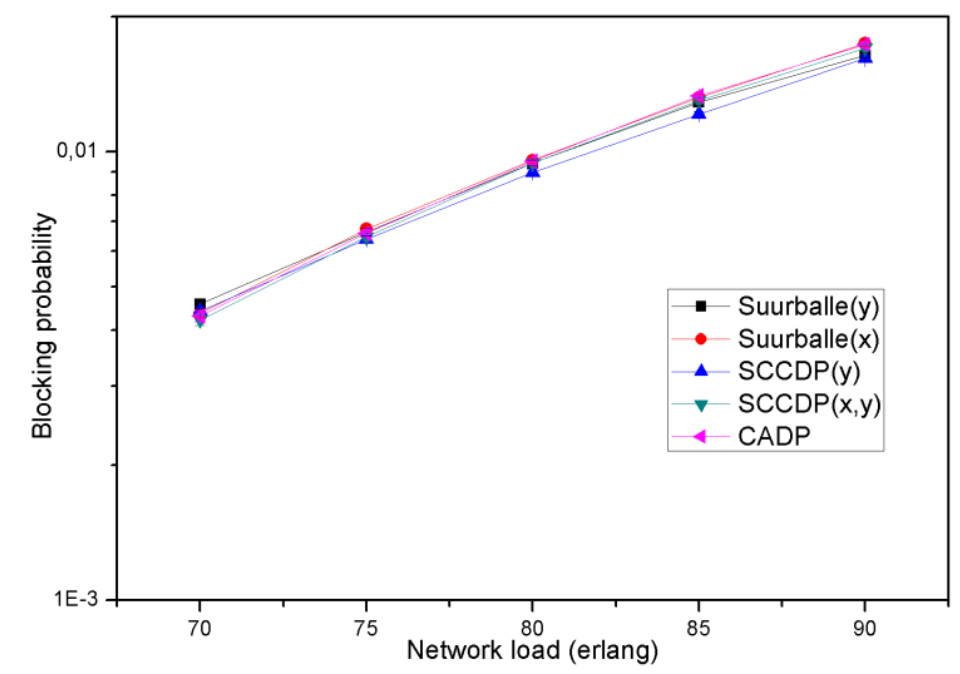

Fig. 5. Comparison of the blocking probability as a function of the network load after 30 different runs and its margin of error at $95 \%$ confidence interval of all investigated DPP algorithms on the Network Topology 2.

\section{CONCLUSIONS}

In this paper, we propose a new dedicated path protection algorithm for EONs, named spectrum continuity and contiguity based dedicated protection (SCCDP). We used two different network topologies in our simulations. We used a simple model based on OSNR that considers ASE noise generation and ASE saturation for physical impairment evaluation. The SCCDP algorithm finds link-disjoint route pairs avoiding the trap topology problem. Each obtained route takes into account the end to end spectrum continuity and contiguity.

We compared our proposal (SCCDP) with other well-known algorithms regarding the blocking probability and throughput, and we showed that in the topology with enough number of link-disjoint routes, the SCCDP algorithm outperformed all other analyzed heuristics. All results demonstrate the importance of taking into account information about the spectrum continuity and contiguity in the dedicated path protection process because it influences the performance of the algorithms severely. 


\section{ACKNOWLEDGMENT}

\section{THE AUTHORS ACKNOWLEDGE THE INSTITUTIONAL SUPPORT FROM UPE AND UFPE, AS WELL AS CNPQ, CAPES AND FACEPE FOR SCHOLARSHIPS AND GRANT.}

\section{REFERENCES}

[1] A. A. M. Saleh and J. M. Simmons, Technology and architecture to enable the explosive growth of the internet, IEEE Commun. Mag. 49(1), 126-132, 2011.

[2] R. Ramaswami and K. N. Sivarajan, Optical Networks: A PracticalPerspective, 3rd ed. San Diego: Morgan Kaufmann, 2009.

[3] G. Zhang, M. De Leenheer, A. Morea and B. Mukherjee, A survey on OFDM-based elastic core optical networking, IEEE Commun. Surv. Tutor. 15(1), 65-87, 2013.

[4] W. Shieh, H. Bao and Y. Tang, Coherent optical OFDM: theory and design, Opt. Express, 16(2), 841-859, 2008.

[5] D. J. Geisler, N. K. Fontaine, R. P. Scott, T. He, L. Paraschis, O. Gerstel, J. P. Heritage and S. J. B. Yoo, Bandwidth scalable, coherent transmitter based on the parallel synthesis of multiple spectral slices using opticalarbitrary waveform generation, Opt. Express, 19(9), 8242-8253, 2011.

[6] S. Ben Yoo, L. Liu, R. Proietti and R. Scott, Software defined elastic optical networking in temporal, spectral, and spatial domains, Photonic Network Communications, 28(1), 19-33, 2014.

[7] S. Bregni, M. Recalcati, F. Musumeci, M. Tornatore and A. Pattavina. Benefits of Elastic Spectrum Allocation in Optical Networks with Dynamic Traffic, IEEE Latin America Transactions, 11, 3642-3648, 2015.

[8] S. Talebi, F. Alam, I. Katib, M. Khamis, R. Salama and G. N. Rouskas, Spectrum management techniques for elastic optical networks: A survey, Optical Switching and Networking, 13, 34-48, 2014.

[9] W. Lu and Z. Zhu, Dynamic service provisioning of advance reservation requests in elastic optical networks, Lightwave Technology, Journal of, 31(10), 1621-1627, 2013.

[10] G. Shen and Hong Guo and Sanjay K. Bose, Survivable elastic optical networks: survey and perspective, Photonic Network Communications, 1(31), 71-87, 2016.

[11] Vivek Alwayn, Optical Network Design and Implementation, 1st ed. Cisco Press, 2004.

[12] Thomas E. Stern, Georgios Ellinas and Krishna Bala, Multiwavelength Optical Networks: Architectures, Design, and Control, 2nd ed. Cambridge University Press, 2009.

[13] E. Horowitz, S. Sahni and S. Rajasekaran, Computer Algorithms, Computer Science Press, Rockville, 1998.

[14] J. W. Suurballe, Disjoint paths in a Network, Networks, 4, 125-145, 1974.

[15] T. Takagi, H. Hasegawa, K. Sato, T. Tanaka, B. Kozicki, Y. Sone and M. Jinno, Algorithms for maximizing spectrum efficiency in elastic optical path networks that adopt distance adaptive modulation, 36th European Conference and Exhibition on Optical Communication, 1-3, 2010.

[16] M. Klinkowski, An Evolutionary algorithm approach for dedicated path protection problem in Elastic Optical Networks, Cybern. Syst., 44 (6), 589-605, 2013.

[17] Jorge Lopez Vizcaíno, Yabin Ye, Víctor Lopez, Felipe Jiménez, Francesco Musumeci, Massimo Tornatore, Achille Pattavina and Peter M. Krummrich, Protection in Optical Transport Networks with Fixed and Flexible Grid: Cost and Energy Efficiency Evaluation, Opt. Switch. Netw., 11, 55-71, 2014.

[18] Krzysztof Walkowiak and Miroslaw Klinkowski and Bartosz Rabiega and Roza Goscien, Routing and spectrum allocation algorithms for elastic optical networks with dedicated path protection, Optical Switching and Networking, 13, 63-75, 2014.

[19] Gangxiang Shen and Yue Wei and Sanjay K. Bose, Optimal Design for Shared Backup Path Protected Elastic Optical Networks Under SingleLink Failure, J. Opt. Commun. Netw. 6, 649-659, 2014.

[20] Seb J. Savory, Congestion Aware Routing in Nonlinear Elastic Optical Networks, IEEE Photonics Tecnology Letters, v. 26, n. 10, 2014.

[21] A. V. S. Xavier, R. C. Almeida, D. A. R. Chaves, C. J. A. Bastos-Filho and J. F. Martins-Filho, Spectrum continuity based routing algorithm for flexible grid optical networks, Microwave and Optoelectronics Conference (IMOC), 2015 SBMO/IEEE MTT-S International, 1-5, 2015.

[22] D. A. R. Chaves, H. A. Pereira, C. J. A. Bastos-Filho and J. F. Martins-Filho, SIMTON: a simulator for transparent optical networks, J. Commun. Inf. Syst. 25(1), 1-10, 2010.

[23] H. A. Pereira, D. A. R. Chaves, C. J. A. Bastos-Filho, and J. F. MartinsFilho, OSNR model to consider physical layer impairments in transparent optical networks, Photonic Netw. Commun., 18, 137-149, 2009.

[24] R. Essiambre, G. Kramer, P.J. Winzer, G.J. Foschini and B. Goebel, Capacity Limits of Optical Fiber Networks, Journal of Lightwave Technology, 28(4), 2010.

[25] Xin Wan, Lei Wang, Nan Hua, Hanyi Zhang and Xiaoping Zheng, Dynamic routing and spectrum assignment in flexible optical path networks, Optical Fiber Communication Conference and Exposition (OFC/NFOEC), 2011 and the National Fiber Optic Engineers Conference, 6-10, 2011.

[26] M. Jinno, B. Kozicki, H. Takara, A. Watanabe, Y. Sone, T. Tanaka and A. Hirano, Distance-adaptive spectrum resource allocation in spectrumsliced elastic optical path network [Topics in Optical Communications], IEEE Communications Magazine, 48(8), 138-145, 2010. 